\title{
Effect of room partitions on airborne and impact sound insulation in large, open rooms
}

\author{
Giovanni Zambon', Roberto Benocci', \\ Fabio Angelini' and Chiara Scrosati ${ }^{2}$
}

\begin{abstract}
ISO 16283 for measuring sound insulation in buildings supersedes ISO I40 but is particularly lacking for measurements in large, open rooms of the type considered here (floor volume: $\sim 4000 \mathrm{~m}^{3}$ ). Following ISO $16283-\mathrm{I}$, the room volume is theoretically divided into smaller volumes through an analysis of sound level distribution. Large absorbing panels in the ceiling prevent the growth of diffuse sound fields, and thus, the analysis makes critical use of normalization factors used in the airborne sound insulation and impact noise levels based on the Sabine equation. Given the likely division of finished floors into rooms, mobile separation walls are used here to investigate the influence of partitions on sound insulation. The results seem to show that they reduce sound insulation and increase impact noise level in each considered enclosed volume. These variations were investigated further through measurements taken in two volumes of different dimensions and at positions near to and far away from the separation walls.
\end{abstract}

\section{Keywords}

Acoustic field measurement, large open rooms, ISO 16283

\section{Introduction}

The method of conducting field measurements of sound insulation in rooms is specified in the ISO 16283 series, ${ }^{1}$ which supersedes the ISO 140 series. ${ }^{2}$ The apparent sound reduction index $\left(R^{\prime}\right)$ (evaluated using equation (1)) is the difference between the average sound pressure level measured in the source room $\left(L_{1}\right)$ and the average sound pressure levels measured in the receiving room $\left(L_{2}\right)$ plus a correction term that is a function of the surface area of the partition between the rooms $(S)$ and the equivalent absorption area $(A)$ which itself depends on the volume of the receiving room $(V)$ and the reverberation time of the receiving room $(T)$ via the Sabine equation: $A=0.16 V / T$. The calculation of $R^{\prime}$ requires measurements of the average sound pressure level in both the source and receiving rooms and measurements of the reverberation time in the receiving room, following the

\footnotetext{
'Department of Earth and Environmental Sciences (DISAT), University of Milano-Bicocca, Milano, Italy
}

${ }^{2}$ Institute for Construction Technologies of the National Research Council (ITC-CNR), Milano, Italy

\section{Corresponding author:}

Giovanni Zambon, Department of Earth and Environmental Sciences (DISAT), University of Milano-Bicocca, Piazza della Scienza I, 20126 Milano, Italy.

Email: giovanni.zambon@unimib.it 
prescription of the ISO 16283 series. Here, the receiving room is the floor directly above the source.

The impact sound pressure level $\left(L_{i}\right)$ is the average sound pressure level in a 1/3-octave band in the receiving room when the floor under test is excited by a standardized impact source. Depending on the correction term applied to the impact sound pressure level, the normalized impact sound pressure level $L_{n}^{\prime}$ (as defined in equation (2)) or the standardized impact sound pressure level $L_{n T}^{\prime}$ (as defined in equation (3)) is obtained. In both cases, the measurements are performed in the receiving room for both the impact sound pressure level $L_{i}$ and the reverberation time $(T)$. Here, the receiving room is the floor directly below the source

$$
\begin{gathered}
R^{\prime}=L_{1}-L_{2}+10 \cdot \log (S / A) \\
L_{n}^{\prime}=L_{i}+10 \cdot \log \left(A / A_{0}\right) \\
L_{n T}^{\prime}=L_{i}-10 \cdot \log \left(T / T_{0}\right)
\end{gathered}
$$

where $A_{0}$ is the reference absorption area (for dwellings $A_{0}=10 \mathrm{~m}^{2}$ ) and $T_{0}$ is the reference reverberation time (for dwellings $T_{0}=0.5 \mathrm{~s}$ ).

In particular, Annex C of ISO 16283-1 includes the prescription of ISO 140-142 (AcousticsGuidelines for Special Situations in the Field). The guidelines are for measurements in rooms of volume not exceeding $250 \mathrm{~m}^{3}$, in which the measurement is characterized by diffused sound fields. The standards do not clearly indicate methodologies for measurements in larger rooms or in rooms where the condition of diffuse sound field is not satisfied.

This issue has not been discussed extensively in the scientific literature. Examples have been discussed defining virtual measurement environments in laboratories, ${ }^{3}$ large rooms characterized by repeating structures, ${ }^{4}$ low-ceilinged and extended industrial environments, ${ }^{5}$ and auditorium and theater design. ${ }^{6-8}$ We considered this issue in a recent study ${ }^{9}$ of airborne and impact sound insulation for large, open rooms, which constitute the floors of a particular building in Milan, Italy. The building, also the subject of this article, consists of nine floors, each of which has similar engineering and architectonic characteristics. In particular, floors from the first floor above the ground floor to the seventh floor above the ground floor are identical, about $3 \mathrm{~m}$ tall (floor to suspended ceiling), with floor areas each of about $1300 \mathrm{~m}^{2}$ and total volumes each of about $3890 \mathrm{~m}^{3}$. Large soundabsorbing panels in the ceiling prevent the growth of diffuse sound fields. Particular attention was paid in the previous work to dividing the entire floor volume into smaller reference volumes, called virtual volumes (VVs), ${ }^{9}$ for analysis. The size of such VVs was found to strongly affect the weighted normalized impact sound pressure level, $L_{n w}^{\prime}$ (see equation (2) and definition of $A$ ). The use of a different correction term, the weighted standardized impact sound pressure level, $L_{n T w}^{\prime}$, excluded the volume dependence.

The values of this single number quantity (SNQ; i.e. $L_{n T w}^{\prime}$ ) were lower than those of the $L_{n w}^{\prime}$ SNQs. $L_{n T w}^{\prime}$ retained some dependence on the VVs, not through the effects of the correction term but by the use of a measurement method that tends to provide higher impact sound levels $\left(L_{2}\right)$ for smaller volumes owing to the tapping machine and microphone generally being closer to each other in a small VV. For the $L_{n w}^{\prime}$ SNQ, the different sizes of the VVs used in the correction term overcome the influence of this latter effect. The dependence of the SNQ $L_{n w}^{\prime}$ on the volume size used for correction also influenced the calculation of the value to be attributed to the entire floor area. The choice of directly using the values obtained from each VV or of using the values from all the measurements extended over the entire volume led to very different $L_{n w}^{\prime}$ results. For $L_{n w}^{\prime}$ 
Table I. Partitioned room characteristics: volumes and floor areas.

\begin{tabular}{lll}
\hline Partitioned volume & Volume $\left(\mathrm{m}^{3}\right)$ & Floor area $\left(\mathrm{m}^{2}\right)$ \\
\hline$V_{1}$ & 1500 & 500 \\
$V_{2}$ & 450 & 150 \\
\hline
\end{tabular}

results from one VV to be extended over the entire volume, it is necessary to identify a measurement procedure that is not affected by the VV size; for example, the calculated values of the $L_{n T w}^{\prime}$ SNQ were consistent regardless of the calculation method. However, for the consideration of $L_{n T w}^{\prime}$ to give meaningful levels, a suitable value for $T_{0}$ is required: $T_{0}$ should be greater than the $0.5 \mathrm{~s}$ currently specified by ISO 16283 when considering reverberation times in large volumes (cf. the Italian standards UNI $8199^{10}$ and $11367^{11}$ ). Considering the airborne sound insulation, $R_{w}^{\prime}$ showed some dependence on VV size for $L_{1}$ levels (levels in the emitter room), the higher levels in the smaller VVs (2 and 4) represent the generally smaller distances between the loudspeaker and the microphone than used in the larger VVs (1 and 3). ${ }^{9}$ Instead, considering the entire volume, the $R_{w}^{\prime}$ SNQ could be deduced from that calculated for a single VV.

\section{Measurement methodology}

The technique of dividing a receiving room into smaller VVs for sound pressure level sampling is

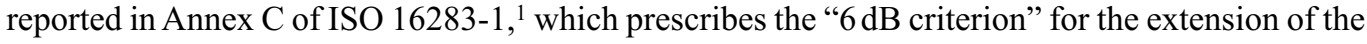
use of such virtual partitions to airborne sound insulation. This criterion considers the identification of VVs characterized by a sound source decay (generally placed in a central position of the $\mathrm{VV})$ of $6 \mathrm{~dB}$.

A detailed description of the determination of the decay of $6 \mathrm{~dB}$ sound pressure levels in the receiving room (i.e. $L_{2}$ ) is given in Zambon et al. ${ }^{9}$ The entire volume was subdivided in smaller volumes (volumes $V_{1}$ and $V_{2}$ ) by oriented strand board (OSB) panels (height, $2.5 \mathrm{~m}$; length, $1.25 \mathrm{~m}$; and thickness, $2.5 \mathrm{~cm}$; Table 1). A 0.5 -m gap remained between the ceiling and the partitions. The panels rested on the floor and were supported vertically by heavy bases. They were placed end to end. This article studies the effect on SNQs of the placement of these mobile OSB walls at the border between volumes $V_{1}$ and $V_{2}$, that is, along the line where a sound pressure level decay of $6 \mathrm{~dB}$ occurred. Given the similar dimensions of the floors, similar volume divisions were adopted for both the source and the receiving rooms. Figure 1 shows the adopted room divisions.

In the superseded ISO 140-14, the 6-dB criterion was indicated only for airborne sound insulation (no indications in the case of impact sound insulation). Currently, only the part about airborne sound insulation is published under ISO 16283, while the other two parts (Part 2 concerning impact sound insulation and Part 3 about façade sound insulation) are under development. In this study, the 6-dB criterion was also used for the evaluation of the decay of the sound pressure level generated by a standard tapping machine.

Figure 2 shows the room partitions of volume $V_{2}$. The sound pressure level was measured on the seventh floor above the source room and on the sixth floor at the positions shown in Figure 3(a) and (b). The impact sound pressure level was measured on the sixth floor at the positions shown in Figure 3(c). The reverberation time was measured on the seventh floor for the airborne sound insulation; the position of the source and microphones are indicated in Figure 3(b). The results were also used for the calculation of impact sound insulation as the two floors are identical. 


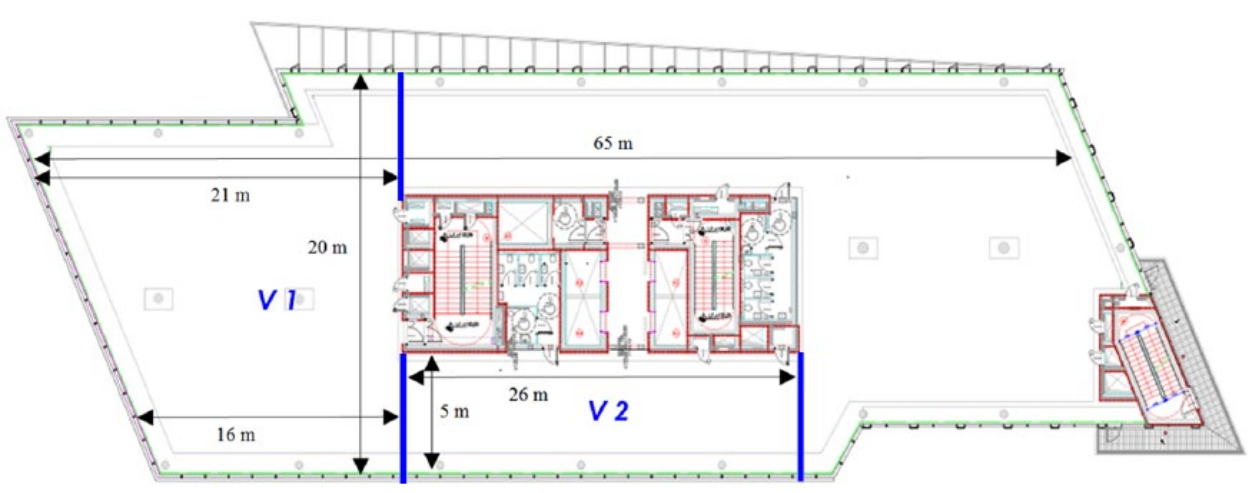

Figure I. Partitioned volumes $V_{1}$ and $V_{2}$ for both the emitting and receiving floors. Oriented strand board (OSB) mobile walls are shown in blue.

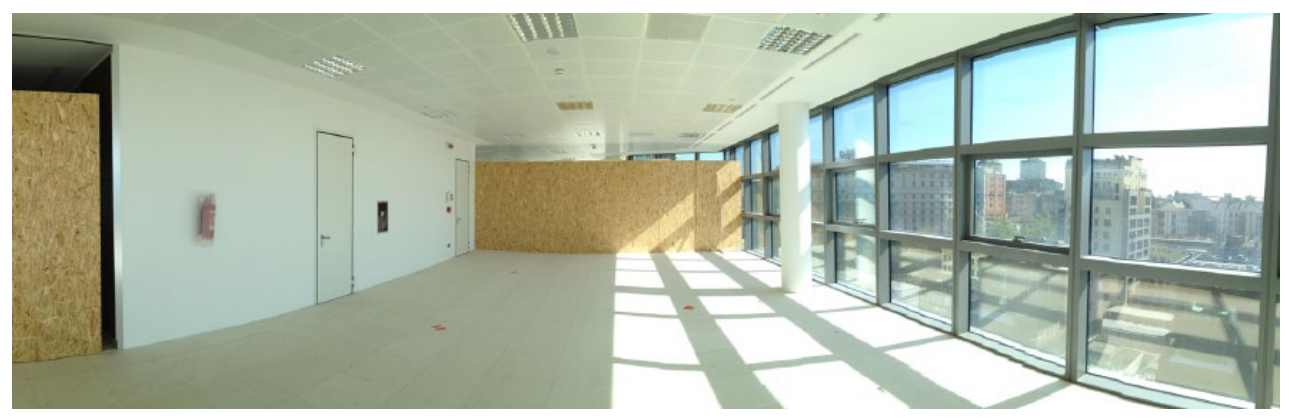

Figure 2. View showing the partial height OSB sheets (center and left) which partition off volume $V_{2}$ from the main volume $V_{1}$ and from the rest of the volume.

\section{Experimental procedure}

Considering the room dimensions and referring to ISO 140-14 (sections A.1 and B.1), the minimum number of positions for the measurement of airborne and impact sound insulation was established for each volume (Tables 2-6).

Measurement positions (microphone positions, source positions, and the distances between the room surfaces and microphones) were chosen to satisfy the minimum distances specified by standard ISO 16283-1 for airborne sound insulation and the superseded ISO 140 Part 7 for impact sound insulation (pending the publication of Part 2 of ISO 16283). To determine the influence of the mobile OSB walls, measurements were repeated both with and without the mobile walls for each microphone and source configuration in volumes $V_{1}$ and $V_{2}$.

\section{Airborne sound insulation results}

Airborne sound insulation measurements were taken with the source on the sixth floor above ground and the receiving room on the seventh. All the presented results are corrected to remove the influence of background noise according to the method described in ISO 16283-1. 


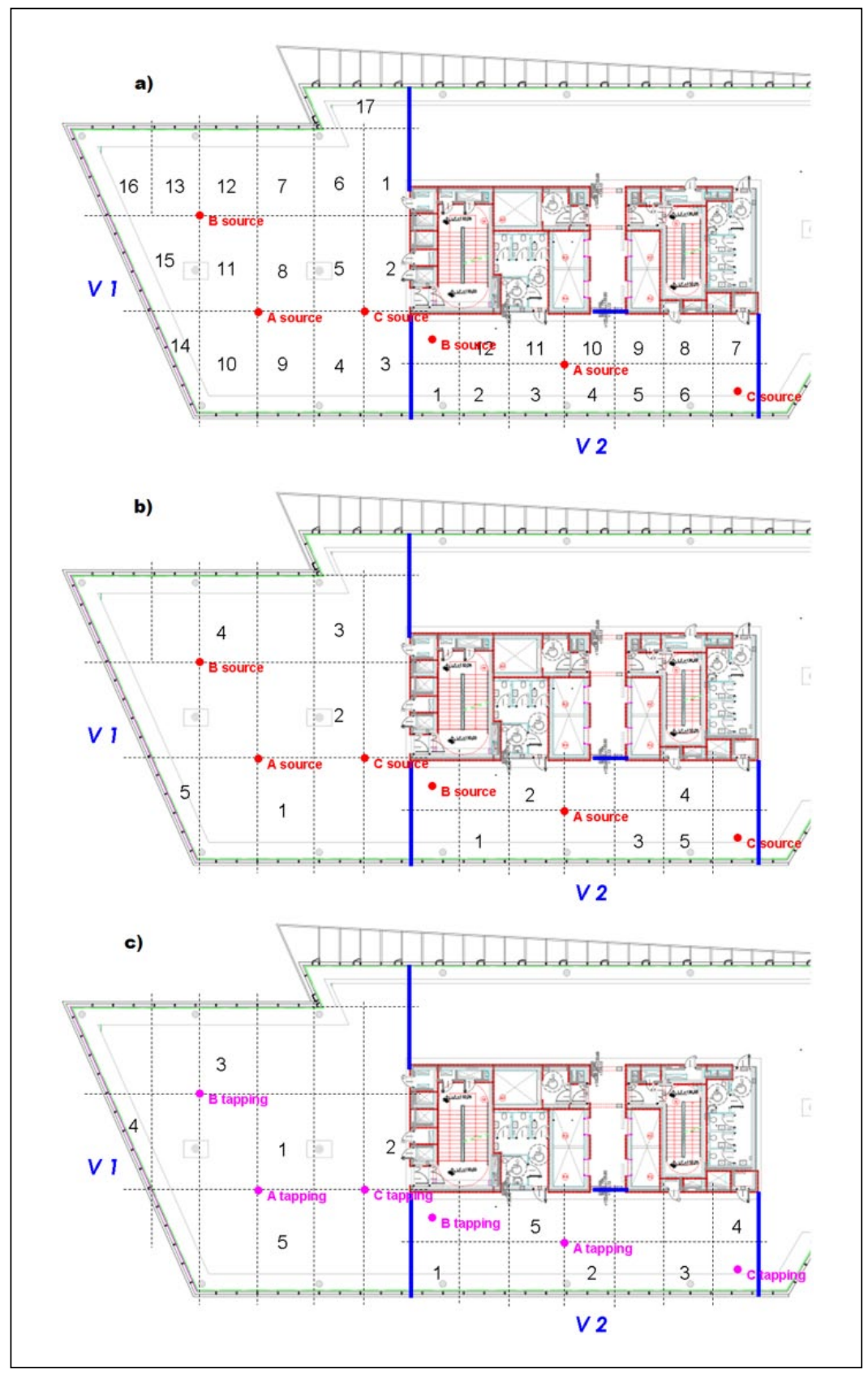

Figure 3. (a) Source (letters from $A$ to $C$ ) and microphone positions (numbers from I to $I 7$ for $V_{1}$ and from $I$ to 12 for $V_{2}$ ) in receiving and source rooms for airborne sound insulation measurements. (b) Source (letters from $A$ to $C$ ) and microphone positions (numbers from I to 5 for both $V_{1}$ and $V_{2}$ ) in the receiving rooms for $T$ measurements for airborne sound insulation measurements. (c) Standard tapping machine (letters from $A$ to $C$ ) and microphone positions (numbers from I to 5 for both $V_{1}$ and $V_{2}$ ) for impact sound insulation measurements. The standard tapping machine refers to the source room and the microphone to the receiving one. 
Table 2. Receiving and source room for airborne and impact sound insulation.

\begin{tabular}{lll}
\hline Measured quantity & Source room & Receiving room \\
\hline Airborne sound insulation & Sixth floor (Figure I (a)) & Seventh floor (Figure I(a)) \\
Impact sound insulation & Seventh floor (Figure I (c)) & Sixth floor (Figure I (c)) \\
Reverberation time & - & Seventh floor (Figure I(b)) \\
\hline
\end{tabular}

Table 3. Number of measurements of the different types used in airborne sound insulation measurements of $V_{1}$.

\begin{tabular}{lllc}
\hline Measured parameter & $\begin{array}{l}\text { Loudspeaker } \\
\text { positions }\end{array}$ & $\begin{array}{l}\text { Measurements at } \\
\text { each position }\end{array}$ & $\begin{array}{l}\text { Total } \\
\text { measurements }\end{array}$ \\
\hline Average sound pressure level, ${ }^{a} L_{1}$ and $L_{2}$ & 3 & 17 & $5 \mathrm{I}$ \\
Background noise, $B$ & - & - & 8 \\
Reverberation time, $T$ & 3 & 5 & $15\left(6^{b}\right)$ \\
\hline
\end{tabular}

aln both the source and the receiving rooms.

bOnly for the case without mobile oriented strand board (OSB) walls.

Table 4. Number of measurements of the different types used in airborne sound insulation measurements of $V_{2}$.

\begin{tabular}{llll}
\hline Measured parameter & $\begin{array}{l}\text { Loudspeaker } \\
\text { positions }\end{array}$ & $\begin{array}{l}\text { Measurements at } \\
\text { each position }\end{array}$ & $\begin{array}{l}\text { Total } \\
\text { measurements }\end{array}$ \\
\hline Average sound pressure levels, ${ }^{a} L_{1}$ and $L_{2}$ & 3 & 12 & 36 \\
Background noise, $B$ & - & - & 6 \\
Reverberation time, $T$ & 3 & 5 & $15\left(6^{b}\right)$ \\
\hline
\end{tabular}

aln both the source and the receiving rooms.

bOnly for the case without mobile oriented strand board (OSB) walls.

Table 5. Number of measurements of the different types used in impact noise level measurements of $V_{1}$.

\begin{tabular}{llll}
\hline Measured parameter & Source positions & $\begin{array}{l}\text { Measurements at } \\
\text { each position }\end{array}$ & $\begin{array}{l}\text { Total } \\
\text { measurements }\end{array}$ \\
\hline Impact sound pressure level, $L_{i}$ & 3 & 5 & 15 \\
Background noise, $B$ & - & - & 3 \\
Reverberation time, $T$ & $3^{\text {a }}$ & 5 & $15\left(6^{b}\right)$ \\
\hline
\end{tabular}

aRefers to loudspeakers.

bOnly for the case without mobile oriented strand board (OSB) walls.

\section{Results for volume $V_{\text {, }}$}

For each of the three loudspeaker positions (A, B, and C in Figure 3), 17 measurement positions of the microphone were chosen (1-17 in Figure 3(a)) for a total of 51 measurements for both $L_{1}$ and $L_{2}$. For each measurement, the $1 / 3$-octave band from 50 to $5000 \mathrm{~Hz}$ was recorded.

Data for $L_{1}$ used in equation (1) provide the apparent sound reduction index $\left(R^{\prime}\right)$ for the receiving room both with and without the mobile walls. 
Table 6. Number of measurements of the different types used in impact noise level measurements of $V_{2}$.

\begin{tabular}{lllc}
\hline Measured parameter & Source positions & $\begin{array}{l}\text { Measurements at } \\
\text { each position }\end{array}$ & $\begin{array}{l}\text { Total } \\
\text { measurements }\end{array}$ \\
\hline Impact sound pressure level, $L_{i}$ & 3 & 5 & 15 \\
Background noise, $B$ & - & - & 3 \\
Reverberation time, $T$ & $3^{\text {a }}$ & 5 & $15\left(6^{b}\right)$ \\
\hline
\end{tabular}

aRefers to loudspeakers.

bOnly for the case without mobile oriented strand board (OSB) walls.

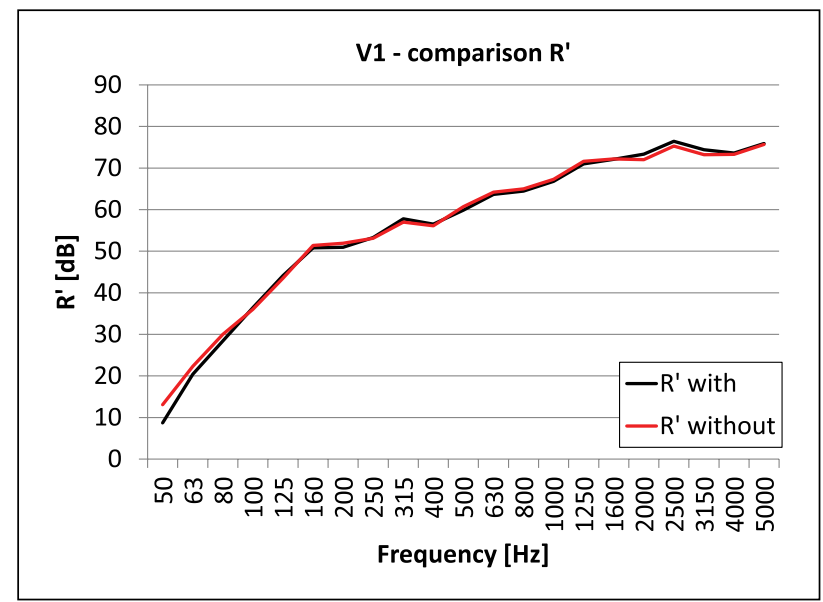

Figure 4. $R^{\prime}$ levels for $V_{1}$ with and without mobile OSB walls.

Apparent sound reduction index $R^{\prime}$. Figure 4 shows the $R^{\prime}$ values calculated for $V_{1}$ with and without the mobile walls. Only small differences are apparent between the two cases, with both showing a weighted apparent sound reduction index $R_{w}^{\prime}$ of $63 \mathrm{~dB}$. In particular, regarding the spectrum adaptation terms $C$ and $C_{\mathrm{tr}}, C$ is the same, whereas $C_{\mathrm{tr}}$ differs by $1 \mathrm{~dB}$ for the two cases ( $-8 \mathrm{~dB}$ with wall separators and $-9 \mathrm{~dB}$ without wall separators).

These small differences are compatible with the measurement uncertainty, in terms of repeatability standard deviation. Repeatability refers to tests performed under conditions that are as constant as possible, with the test performed during a short interval of time in one laboratory by one operator using the same equipment. In this case, it is therefore possible to refer to repeatability standard deviation, as the measurements were performed by the same operators with the same equipment in the same location. The repeatability standard deviation for SNQs for airborne sound insulation is described in ISO 12999-1.12 In this standard, the uncertainty refers in general to airborne sound insulation without making a distinction between airborne sound insulation of floors or walls. A recent study on the airborne sound insulation uncertainty, ${ }^{13}$ evaluated by a Round Robin Test (RRT), has highlighted that the uncertainties of lightweight samples are lower than the uncertainties of heavy types of construction. Considering that the operators who carried out the measurement in this study have participated in the RRT and that in that paper the uncertainties for wall and floor were calculated separately, Table 7 shows the repeatability standard deviation of ISO 12999-1 and the repeatability standard deviation coming from the RRT for the floor. 
Table 7. Repeatability standard deviation for $R_{w}^{\prime}$ and $R_{w}^{\prime}$ with the spectrum adaptation terms $C$ and $C_{\text {tr }}$ referring to $\mathrm{RRT}^{13}$ and ISO $12999-$ I. $^{12}$

\begin{tabular}{llll}
\hline Reference & \multicolumn{4}{l}{ Repeatability standard deviation $(\mathrm{dB})$} \\
\cline { 2 - 4 } & $R_{w}^{\prime}$ & $R_{w}^{\prime}+C$ & $R_{w}^{\prime}+C_{\mathrm{tr}}$ \\
\hline RRT $^{13}$ & 0.5 & 0.6 & 0.9 \\
ISO I2999-| 12 & 0.4 & 0.5 & 0.7 \\
\hline
\end{tabular}

RRT: Round Robin Test.

Table 8. Values of $R_{w}^{\prime}$ with spectrum adaptation terms for $V_{1}$, with and without mobile walls, calculated with I dB or $0.1 \mathrm{~dB}$ steps.

\begin{tabular}{|c|c|c|c|c|}
\hline \multirow[t]{2}{*}{ Configuration } & \multirow{2}{*}{$\frac{\mathrm{IdB} \text { step }}{R_{w}^{\prime}\left(C ; C_{\mathrm{tr}}\right)}$} & \multicolumn{3}{|c|}{$0.1 \mathrm{~dB}$ steps } \\
\hline & & $R_{w}^{\prime}$ & $R_{w}^{\prime}+C$ & $R_{w}^{\prime}+C_{\mathrm{tr}}$ \\
\hline With mobile walls & $63(-2 ;-8)$ & 63.6 & 60.9 & 54.5 \\
\hline Without mobile walls & $63(-2 ;-9)$ & 63.7 & 60.8 & 54.2 \\
\hline
\end{tabular}

Comparing the results obtained in Table 8 with the uncertainties of Table 7, it is evident that the differences between the configuration with and without the mobile OSB walls fall within the limits of the measurement uncertainty of measurement, and therefore, the mobile OSB walls do not influence the measurement.

Average sound pressure levels measured in the receiving room $L_{2}$. Measurements for $L_{2}$ were similar in each case (Figure 5), especially at central frequencies. These results suggest that the presence of the room separators does not affect $L_{2}$. When measuring the sound pressure level in rooms, it is the spatial average value that is required. In this kind of measurements, it is necessary to consider temporal as well as spatial averaging. Considering the spatial variation of the sound pressure level, it is generally assumed that the uncertainty due to time-averaging of the random noise signal at each position is negligible. Therefore, to have an idea of the magnitude of the differences between the configuration with and without separation walls, the standard deviation calculated from all the microphone positions was added to the $L_{2}$ average value as error bars in the graph of Figure 5 .

Reverberation time. Reverberation time $T$ was longer without separation walls than with them. This can be explained, taking into account the differences of the volumes involved (Figure 6).

In addition, it is useful to note that the absorption coefficient curve of the false ceiling material presents a high absorption value $(\alpha=0.8)$ at $250 \mathrm{~Hz}(1 / 1$-octave band). This characteristic may explain that at such frequencies, the reverberation time is the same for both cases with and without separation walls.

Applying the Sabine formula $(T=0,16 \cdot(V / A))$ to the two cases $V$ tot and $V_{1}$ with mobile OSB walls to estimate the reverberation time, we obtained the results reported in Table 9:

These values are very different from those presented in Figure 6. To investigate this difference, the reverberation time within the volume $V_{1}$ with and without the OSB walls was estimated by means of a commercial software for the simulation of room acoustics (Ramsete). ${ }^{15,16}$ This software uses algorithms also suitable to non-Sabinian sound fields. Calculations were performed in octave bands, using the absorption coefficient at $250 \mathrm{~Hz}$ band of $\alpha=0.8$. 


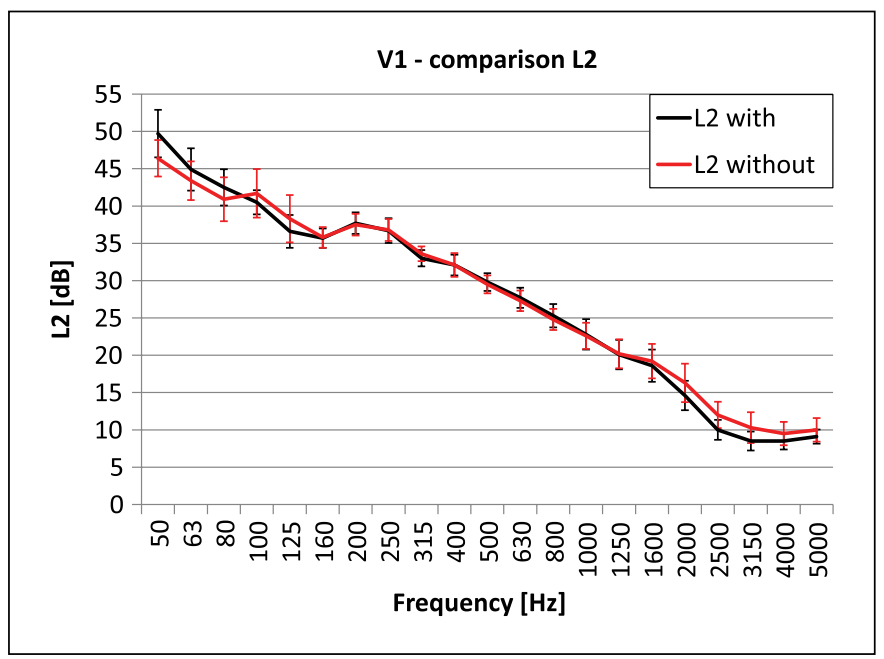

Figure 5. $L_{2}$ levels for $V_{1}$ with (black) and without (red) OSB walls; the error bars are the standard deviation calculated from all the microphone positions.

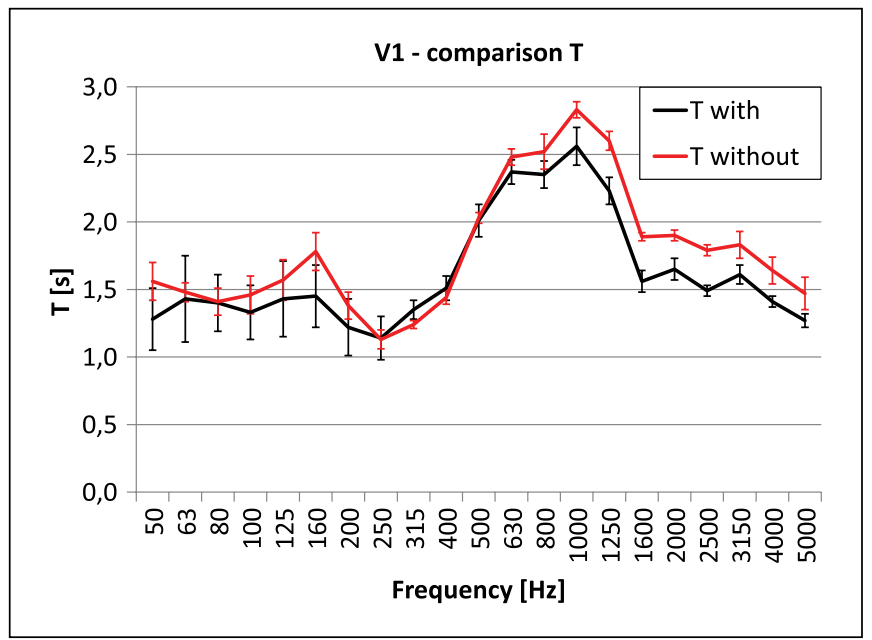

Figure 6. Reverberation time $T$ for $V_{1}$ with (black) and without (red) wall separators the error bars are the standard deviation calculated from all the microphone's positions.

In Figures 7 and 8, the scheme adopted for the simulation is presented.

The results obtained with the simulation model for the positions illustrated in Figures 7 and 8 are shown in Table 10.

The simulation results show values in agreement with the experimental measurement in confirming that the sound field in $V_{1}$ is non-diffuse. On the other hand, this could be inferred by the constrains imposed by the use of Sabine relation: first, the three dimensions of the room must be mutually comparable (here the height is much smaller than the other two), and second, the sound absorption coefficient of the walls should be on average the same in the entire room (in our case, we have all reflective surfaces with the exception of a very absorbent ceiling). ${ }^{5}$ 
Table 9. Calculation of the reverberation time by means of the Sabine formula.

$\operatorname{Vtot}\left(\mathrm{m}^{3}\right)$

$S$ glass facades $\left(\mathrm{m}^{2}\right)$

$S$ plasterboard walls $\left(\mathrm{m}^{2}\right)$

$S$ floor panels $\left(\mathrm{m}^{2}\right)$

1300

$S$ acoustic ceiling $\left(\mathrm{m}^{2}\right)$

1300

$\mathrm{T} 250 \mathrm{~Hz}(\mathrm{~s})$

$V_{1}\left(\mathrm{~m}^{3}\right)$ 1500

$S$ glass facades $\left(\mathrm{m}^{2}\right)$ 168

$S$ plasterboard walls $\left(\mathrm{m}^{2}\right)$

$S$ floor panels $\left(\mathrm{m}^{2}\right)$

$S$ acoustic ceiling $\left(\mathrm{m}^{2}\right)$

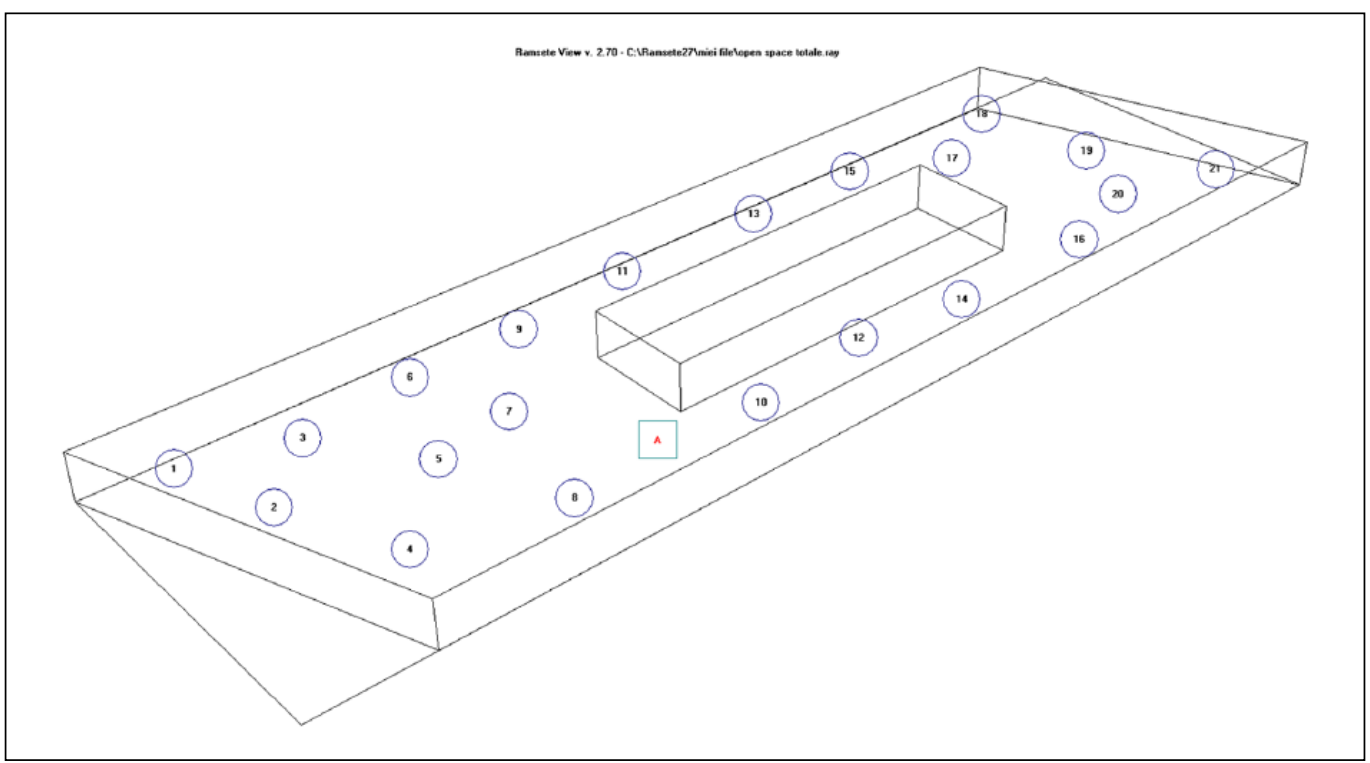

Figure 7. Sketch of the room without mobile OSB walls. Shown are the 20 receptors and the source (A) placed in $V_{1}$.

\section{Results for volume $V_{2}$}

Measurements in $V_{2}$ have the source in three different positions and the microphone in 12 positions for each source position, giving a total of 36 measurements for both $L_{1}$ and $L_{2}$ (see Figure 3(a)).

Apparent sound reduction index $R^{\prime}$. The calculated weighted apparent sound reduction index in the case of $V_{2}$ gives $R_{w}^{\prime}=65 \mathrm{~dB}$ for the bare room and $64 \mathrm{~dB}$ for the room with wall separators (Table 11). The spectrum adaptation terms $C$ and $C_{\mathrm{tr}}$ are the same in both cases: $C=-2 \mathrm{~dB}$ and $C_{\mathrm{tr}}=-7 \mathrm{~dB}$. The results in Figure 9 show that, excluding low frequencies, $R^{\prime}$ is higher in the case of the bare room. 


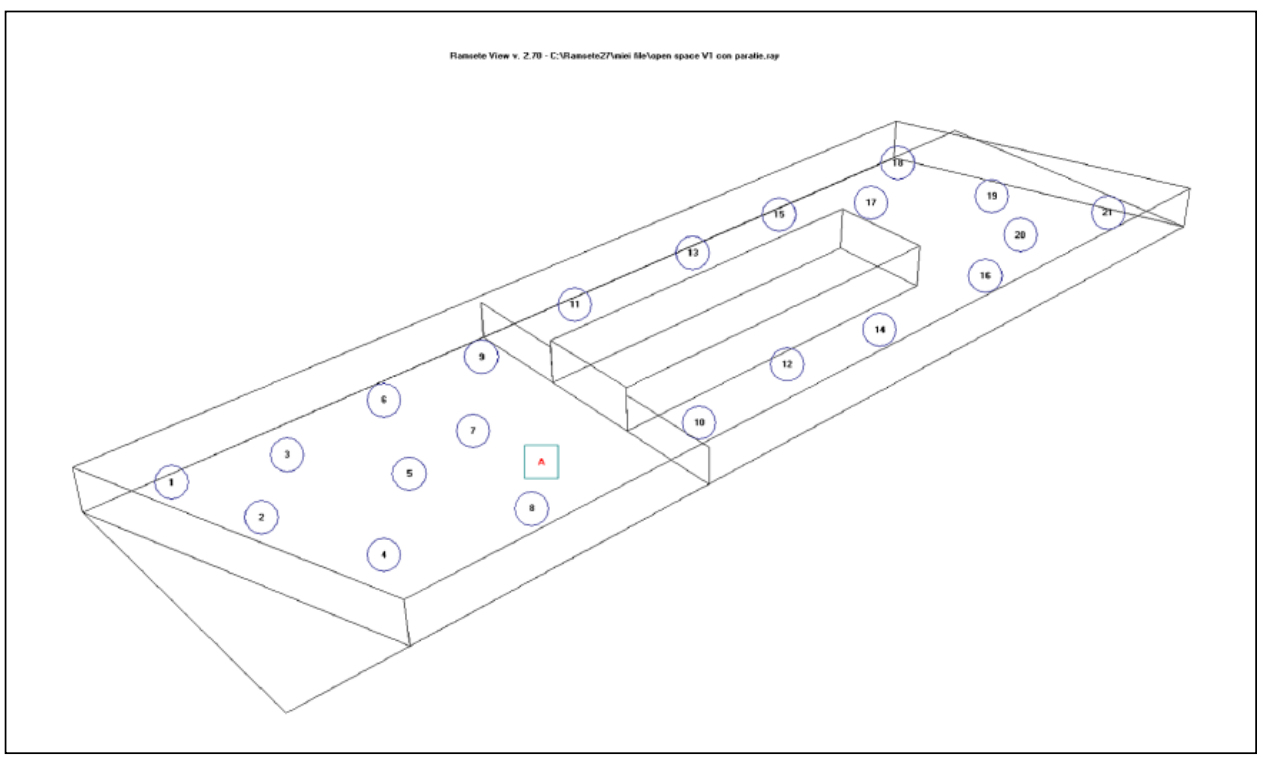

Figure 8. Sketch of the room with mobile OSB walls. Shown are the 20 receptors and the source (A) placed in $V_{1}$.

Table 10. Values of reverberation time (at $250 \mathrm{~Hz}$, octave band) for 20 positions within the room.

\begin{tabular}{llllllllllllllllllllllll}
\hline OSB & Position & 1 & 2 & 3 & 4 & 5 & 6 & 7 & 8 & 9 & 10 & 11 & 12 & 13 & 14 & 15 & 16 & 17 & 18 & 19 & 20 \\
\hline Without & $\mathrm{T} 30 \mathrm{~s}$ & 1.21 & 1.2 & 1.26 & 1.21 & 1.23 & 1.22 & 1.17 & 1.17 & 1.18 & 1.21 & 1.23 & 1.23 & 1.16 & 1.28 & 1.23 & 1.39 & 1.24 & 1.22 & 1.29 & 1.38 \\
With & $\mathrm{T} 30 \mathrm{~s}$ & 0.99 & 1 & 1.02 & 0.99 & 1.01 & 1.02 & 1 & 1.02 & 1 & 1.22 & 1.1 & 1.16 & 1.1 & 1.16 & 1.16 & 1.24 & 1.23 & 1.2 & 1.3 & 1.26
\end{tabular}

OSB: oriented strand board.

The first 9 are placed in the volume $V_{1}$.

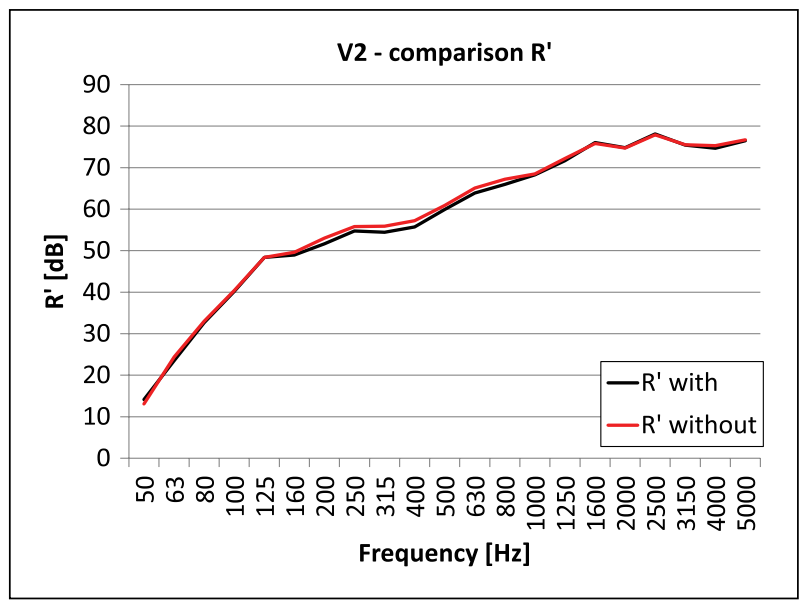

Figure 9. $R^{\prime}$ for $V_{2}$ with and without mobile OSB walls. 


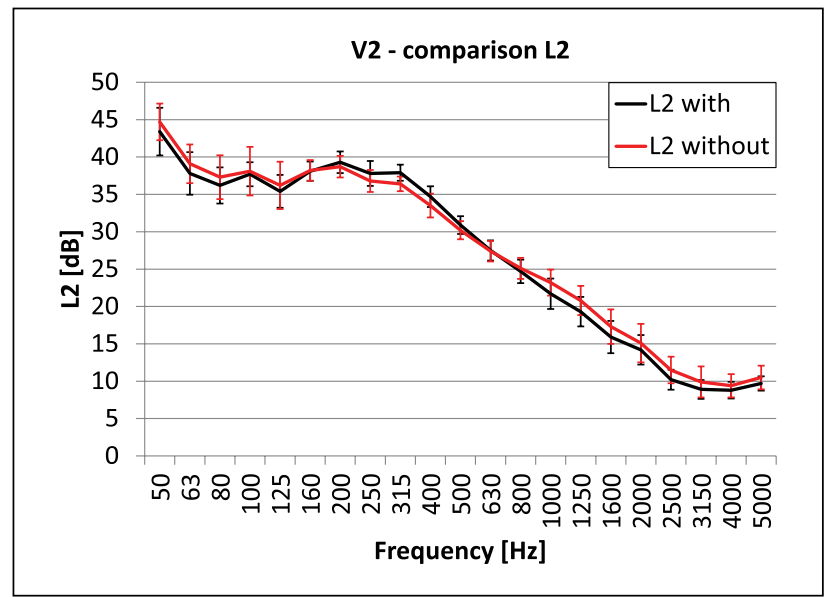

Figure 10. $L_{2}$ levels for $V_{2}$ with (black) and without (red) wall separators; the error bars are the standard deviation calculated from all the microphone's positions.

Table I I. Values of $R^{\prime}{ }_{w}$ with spectrum adaptation terms for $V_{2}$, with and without mobile walls, calculated with I dB or $0.1 \mathrm{~dB}$ steps.

\begin{tabular}{|c|c|c|c|c|}
\hline \multirow[t]{2}{*}{ Configuration } & \multirow{2}{*}{$\frac{\mathrm{I} \mathrm{dB} \text { step }}{R_{w}^{\prime}\left(C ; C_{\mathrm{tr}}\right)}$} & \multicolumn{3}{|c|}{$0.1 \mathrm{~dB}$ steps } \\
\hline & & $R_{w}^{\prime}$ & $R_{w}^{\prime}+C$ & $R_{w}^{\prime}+C_{\mathrm{tr}}$ \\
\hline With mobile walls & $64(-2 ;-7)$ & 64.2 & 62.2 & 56.9 \\
\hline Without mobile walls & $65(-2 ;-7)$ & 65.2 & 63.0 & 57.5 \\
\hline
\end{tabular}

In this case, the differences with and without mobile walls are greater than the measurement uncertainty. In fact in this case, the major differences are in the central region of frequencies, the region that influences the SNQ, while in the previous case $\left(V_{1}\right)$, the major differences were found in the low-frequency region, which does not influence the SNQ. It is therefore interesting to evaluate the differences due to the measurement positions and reverberation time measurements.

Average sound pressure levels measured in the receiving room $L_{2}$. Contrary to expectation, Figure 10 shows that $L_{2}$ values are lower in the open room than the divided room for the central frequencies $(200-630 \mathrm{~Hz})$.

Reverberation time. Such behavior cannot be explained by the reverberation time measurements, $T$ (Figure 11), because they present higher values without the wall separators (as expected because there is a larger volume). As a consequence, the apparent sound reduction index, $R^{\prime}$, is higher without the mobile walls.

Similar to the treatment of $V_{1}$, values of $L_{2}$ for $V_{2}$ were measured at varying distance from the walls to highlight their influence.

$L_{2}$ evaluation with respect to measuring position (distance from separation walls). Figure 12 compares the values of $L_{2}$ measured with and without mobile walls. The first graph shows data from near the walls at positions 1, 7, and 10 in Figure 3(a); the second graph considers measurements further from the walls at positions 5, 6, 8, 9, 11, and 12 in Figure 3(a). 


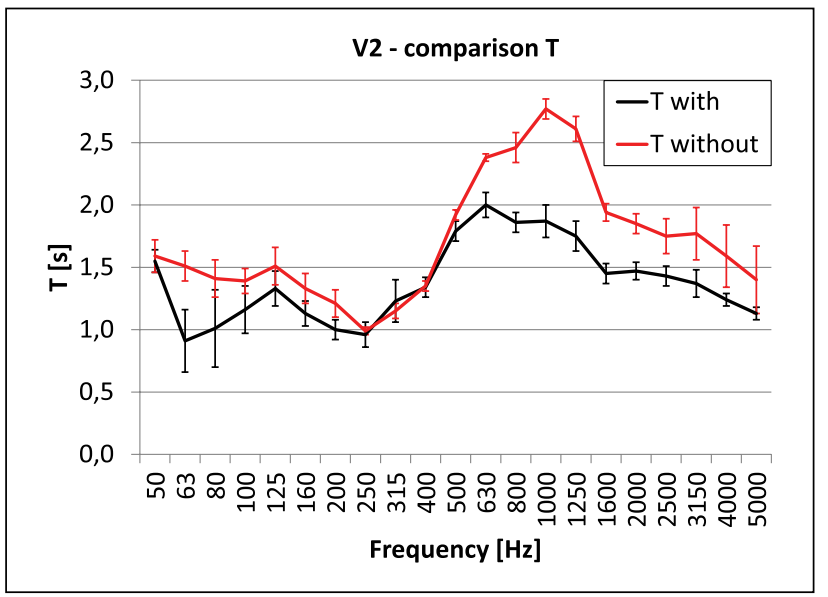

Figure I I. Reverberation time $T$ for $V_{2}$ with and without wall separators; the error bars are the standard deviation calculated from all the microphone's positions.

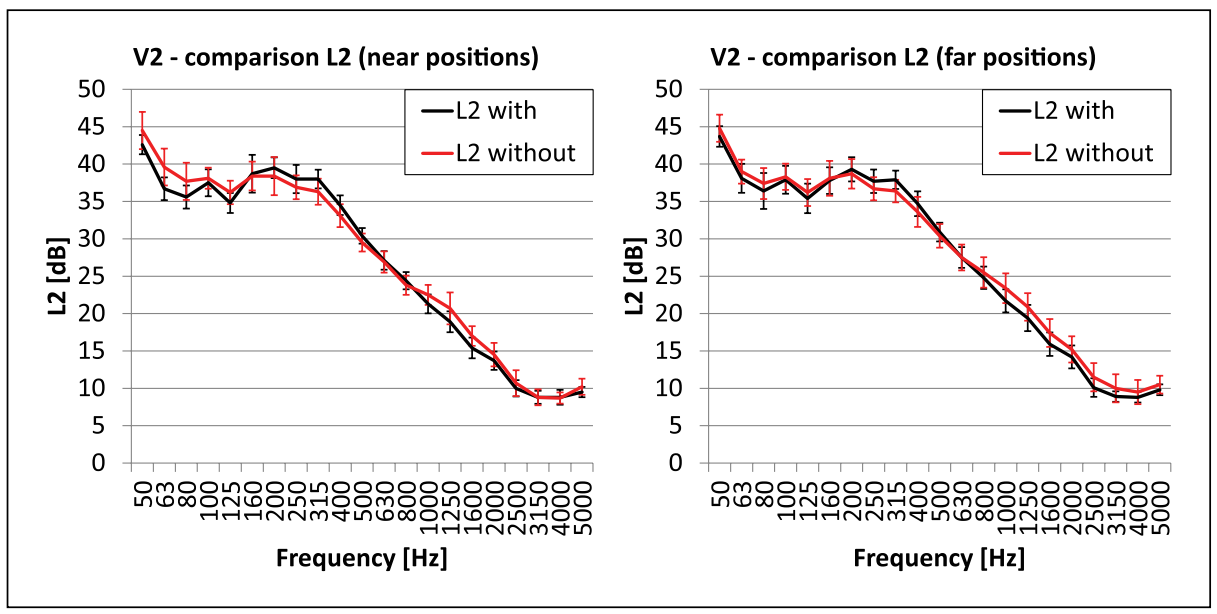

Figure 12. $L_{2}$ levels for $V_{2}$ with and without mobile walls measured at positions (first graph) near to and (second graph) far from the walls; the error bars are the standard deviation calculated from all the microphone's positions.

Because it is supposed that the increase in noise level in the presence of the mobile OSB walls is due to reflections of the walls (an effect more apparent near the walls), the influence of the presence of the separation walls will be investigated by comparing data measured at positions of varying distance from the walls.

To check whether the difference between the two average $L_{2}$ profiles with and without separation walls is statistically significant, the Mann-Whitney-Wilcoxon test was performed as for volume $V_{2}$. This is a robust non-parametric test which is well suited to data on which linear, exponential, or logarithmic functions are applied. Band frequencies showing significant differences (independence) at $\alpha=0.05$ significance level (green dotted line) are shown in Figure 13. Results show that there are a significant number of bands presenting significant differences at the level of significance $\alpha=0.05$. 


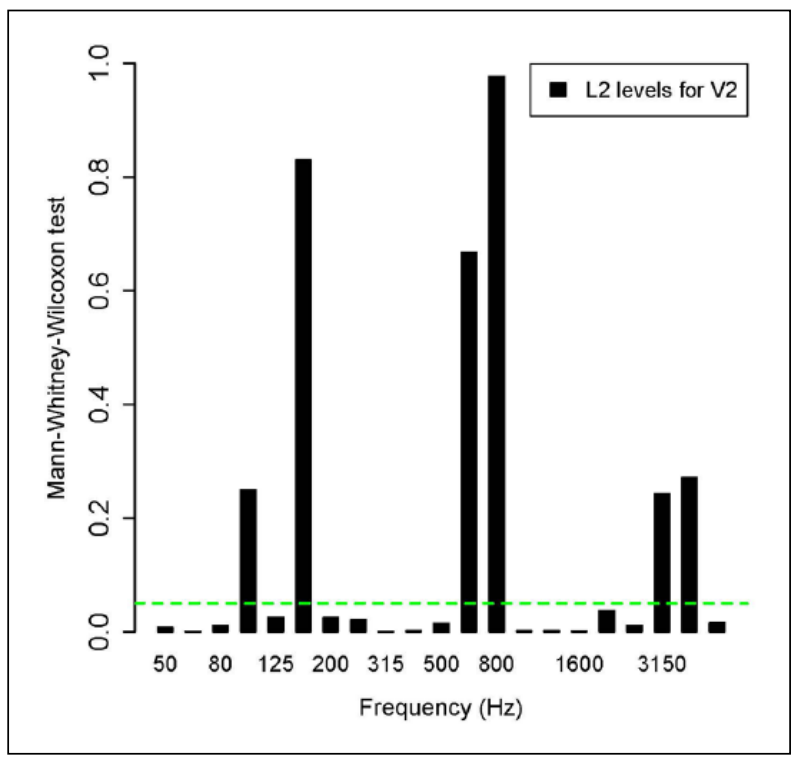

Figure 13. Mann-Whitney-Wilcoxon test performed as for volume $V_{2}$. Band frequencies showing significant differences (independence) are placed below the significance threshold level $\alpha=0.05$ (green dotted line).

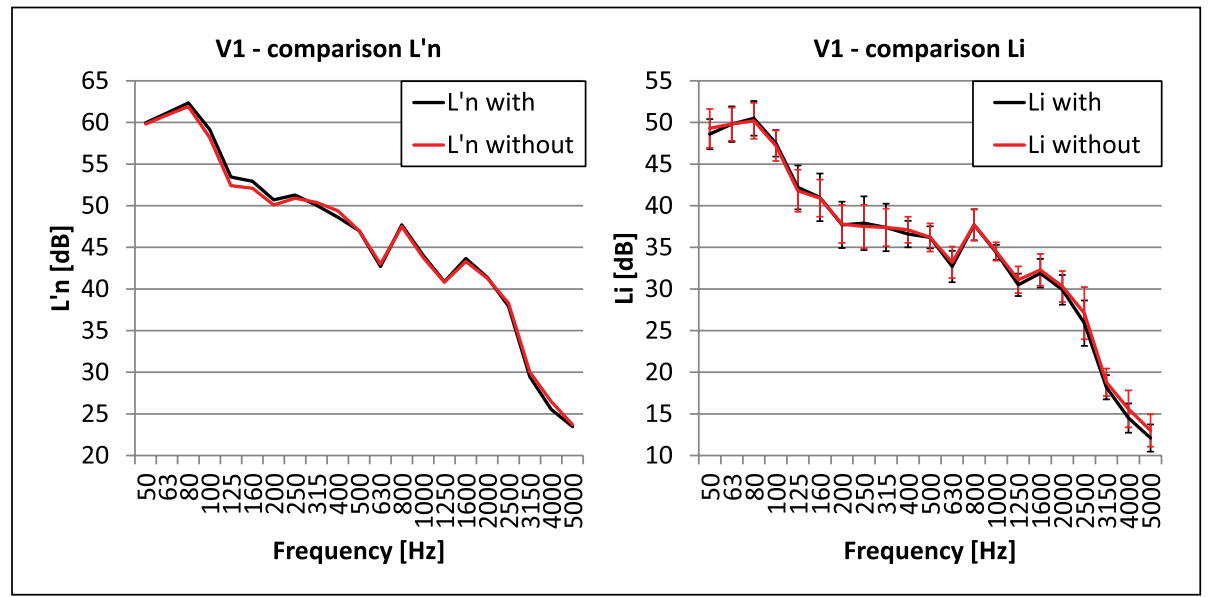

Figure 14. $L_{n}^{\prime}$ (left) and $L_{i}$ (right) levels for volume $V_{1}$ with and without mobile OSB walls; the error bars are the standard deviation calculated from all the microphone's positions.

At positions close to the mobile walls, $L_{2}$ values are greater in the presence of walls than in their absence for the frequencies $160-630 \mathrm{~Hz}$ and are similar for the other frequencies (see Figure 12). This is not justifiable considering that the reverberation times are lower for almost all frequencies (see Figure 11). A further confirmation of the influence of the presence of the mobile walls on $L_{2}$ is given in Figure 12: the values with and without mobile walls measured at positions far from the walls show some small differences that are less than those measured near the walls. 
Table 12. Values of $R^{\prime}{ }_{w}$ for $V_{2}$ using measurements at position far and near the walls in cases with and without mobile partition walls.

\begin{tabular}{|c|c|c|}
\hline & $V_{2}$, near positions & $V_{2}$, far positions \\
\hline & $R_{w}^{\prime}\left(C ; C_{\mathrm{tr}}\right)$ & $R_{w}^{\prime}\left(C ; C_{\mathrm{tr}}\right)$ \\
\hline Without walls & $65(-2 ;-7)$ & $65(-2 ;-8)$ \\
\hline With walls & $64(-2 ;-7)$ & $64(-2 ;-7)$ \\
\hline
\end{tabular}

Table 13. $L_{n w}^{\prime}$ SNQs for volumes $V_{1}$ and $V_{2}$ with and without mobile walls.

\begin{tabular}{lll}
\hline & $V_{1}, L_{n w}^{\prime}\left(C_{i}\right)$ & $V_{2}, L_{n w}^{\prime}\left(C_{i}\right)$ \\
\hline Without walls & $48(-2)$ & $45(-2)$ \\
With walls & $49(-1)$ & $47(-2)$ \\
\hline
\end{tabular}

SNQ: single number quantity.

Because of the differences in the $L_{2}$ levels, the values of the weighted apparent sound reduction index in the presence of mobile OSB walls, for both far and near positions, are $1 \mathrm{~dB}$ lower than those in the absence of the walls (Table 12).

\section{Impact sound insulation}

Impact sound pressure level was measured by placing a standard tapping machine on the seventh floor and the microphones on the sixth floor. All the presented results are corrected to remove the influence of background noise, according to the method described in ISO 16283-1. ${ }^{1}$

\section{Results for $V_{1}$ and $V_{2}$}

The SNQ $L_{n w}^{\prime}$ values are always higher in the presence of the mobile walls than in their absence and are $2 \mathrm{~dB}$ lower in the smaller volume $\left(V_{2}\right)$ than in the larger volume $\left(V_{1}\right)$ (see Table 13). Figures 14 and 15 show the values of $L_{n}^{\prime}$ and $L_{2}$ for the two volumes. The differences are due to the impact sound pressure levels $\left(L_{i}\right)$ in the receiving room being always higher in the presence of the mobile walls than in their absence, even if the corresponding reverberation times are lower (see Figures 6 and 11).

As in the case of airborne sound insulation, the differences are significant in the case of volume $V_{2}$.

These differences are also evaluated for the standardized impact sound pressure level $L_{n T}^{\prime}$. Table 14 lists the values of $L_{n T w}^{\prime}$ for volumes $V_{1}$ and $V_{2}$ with and without the mobile walls, calculated moving the reference curve in $1 \mathrm{~dB}$ or $0.1 \mathrm{~dB}$ steps. ${ }^{14}$ The latter case enhances the differences and makes them prominent, while the former case does not.

As previously reported, ${ }^{9}$ for an open plan office, $L_{n T}^{\prime}$ provides values much lower than $L_{n}^{\prime}$. This confirms that for large volumes, it would be more appropriate to use a higher reference reverberation time than that currently defined for dwellings $\left(T_{0}=0.5 \mathrm{~s}\right)$ (see also the considerations mentioned in section 'Reverberation time').

As for airborne sound insulation, the small differences in the SNQs values for volume $V_{1}$ are compatible with the measurement uncertainty. The repeatability standard uncertainty of SNQs for 


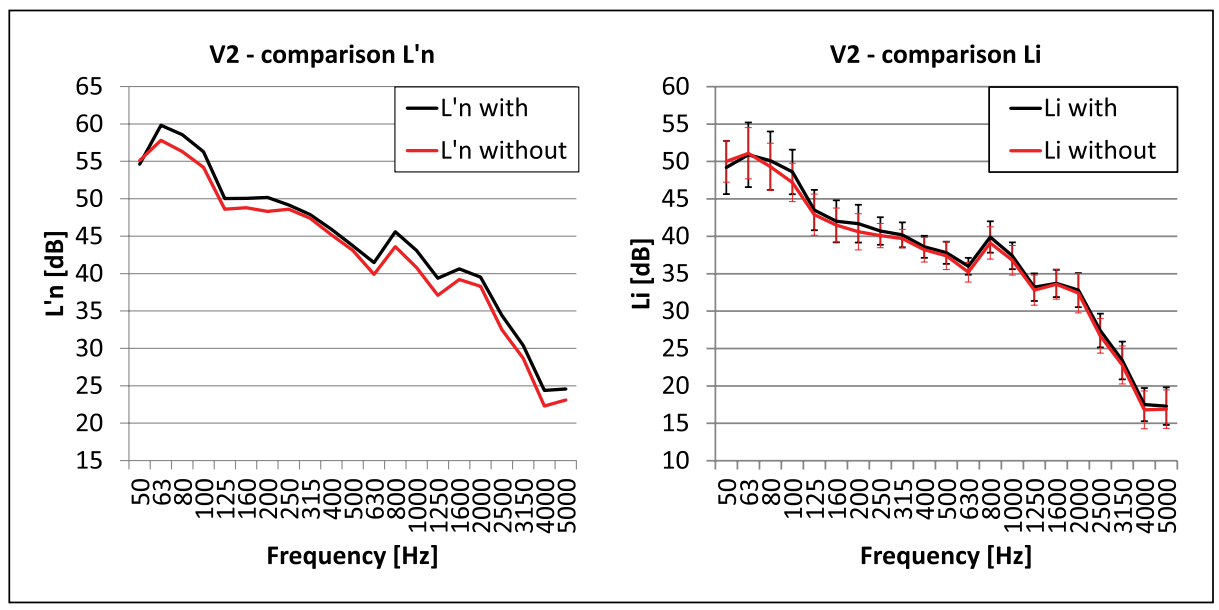

Figure 15. $L_{n}^{\prime}$ (left) and $L_{i}$ (right) levels for volume $V_{2}$ with and without mobile OSB walls; the error bars are the standard deviation calculated from all the microphone's positions.

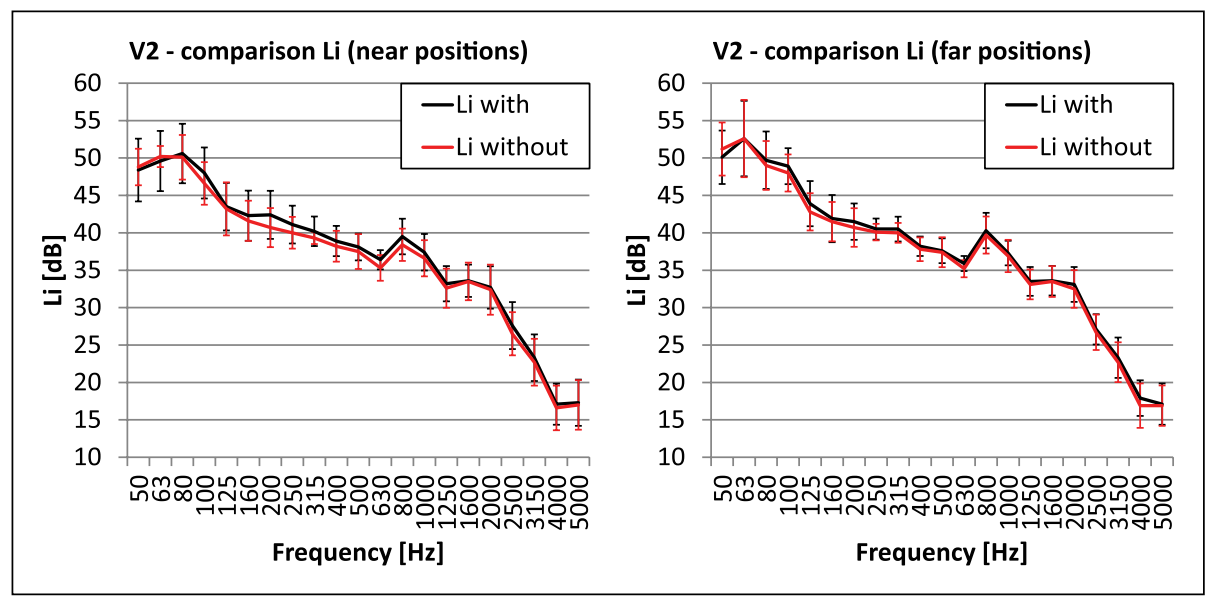

Figure 16. $L_{i}$ measured with and without mobile walls. (First graph) Measurement close to the wall at points I and 4. (Second graph) Measurement far from the wall at points 3 and 5; the error bars are the standard deviation calculated from all the microphone's positions.

Table 14. Values of $L_{n T w}^{\prime}$ for $V_{1}$ and $V_{2}$ with and without mobile walls, calculated with I dB or $0.1 \mathrm{~dB}$ steps.

\begin{tabular}{lll}
\hline & $V_{1}, L_{n T w}^{\prime}$ & $V_{2}, L_{n T w}^{\prime}$ \\
\hline Without mobile walls (I dB steps) & 32 & 34 \\
With mobile walls (I dB steps) & 32 & 35 \\
Without mobile walls (0.I dB steps) & 31.1 & 33.2 \\
With mobile walls (0.I dB steps) & 31.5 & 34.6 \\
\hline
\end{tabular}


Table 15. Values of $L_{n w}^{\prime}$ and $L_{n T w}^{\prime}$ SNQs for $V_{2}$ using measurements taken far from and near to the walls calculated using I dB or $0.1 \mathrm{~dB}$ steps.

\begin{tabular}{|c|c|c|c|c|}
\hline & $V_{2}$, near positions & $V_{2}$, far positions & $V_{2}$, near positions & $V_{2}$, far positions \\
\hline & $L_{n w}^{\prime}\left(C_{i}\right)$ & $L_{n w}^{\prime}\left(C_{i}\right)$ & $L_{n T w}^{\prime}\left(C_{i}\right)$ & $L_{n T w}^{\prime}\left(C_{i}\right)$ \\
\hline $\begin{array}{l}\text { Without mobile walls } \\
\text { (I dB steps) }\end{array}$ & $45(-2)$ & $45(-1)$ & $33(-1)$ & $34(-2)$ \\
\hline $\begin{array}{l}\text { With mobile walls } \\
\text { (I dB steps) }\end{array}$ & $47(-2)$ & $47(-2)$ & $35(-1)$ & $35(-1)$ \\
\hline $\begin{array}{l}\text { Without mobile walls } \\
(0.1 \text { dB steps) }\end{array}$ & 44.6 & 44.9 & 33.0 & 33.3 \\
\hline $\begin{array}{l}\text { With mobile walls } \\
(0.1 \mathrm{~dB} \text { steps })\end{array}$ & 46.2 & 46.2 & 34.7 & 34.6 \\
\hline
\end{tabular}

SNQ: single number quantity

impact sound insulation laid down in ISO $12999-1^{12}$ is equal to $0.5 \mathrm{~dB}$ and equal to $0.6 \mathrm{~dB}$ for SNQs plus the spectrum adaptation term $C_{i}$. Again the differences found for volume $V_{2}$ are greater than the standard uncertainty magnitude.

As for the measurements of apparent sound reduction index, the impact sound pressure level measurements were also influenced by the mobile walls. The influence on $L_{i}$ was more significant when the measuring points were close to the mobile walls.

\section{Evaluation of $L_{i}$ with respect to measuring position (distance from mobile walls)}

Figure 16 compares values of $L_{i}$ with and without the mobile walls, measured close to the walls (in positions 1 and 4, left graph) and far from the walls (in positions 3 and 5,right graph).

Table 15 lists the values of the SNQs $L_{n w}^{\prime}$ and $L_{n T w}^{\prime}$ for $V_{2}$ using measurements both near to and far from the walls. The two SNQs were calculated using steps of $0.1 \mathrm{~dB}$.

Table 15 clearly shows that the SNQ values of the impact sound insulation are always higher in the presence of the mobile walls than in their absence. Analysis of the $L_{n T w}^{\prime}$ SNQ shows that this difference is reduced at distances further from the mobile walls; this is confirmed by the results obtained using $0.1 \mathrm{~dB}$ steps.

\section{Conclusion}

The international standards are poor in their concern for the measurement of airborne sound insulation and impact sound insulation in large, open rooms. Given that this type of environment is increasingly popular (e.g. open plan offices) and that the requirements of acoustic comfort are increasing, the production of specific standards is desirable. This work aims to provide an initial contribution to identify some of the factors that must be covered in the drafting of these standards. Measurements were carried out in a modern building that will be dedicated exclusively to offices. In a previous work, ${ }^{9} \mathrm{SNQs}$ were calculated by dividing the open floor (i.e. a single room) into smaller volumes, where the criterion of $6 \mathrm{~dB}$ decay was satisfied. The results clearly indicated that the SNQ values obtained depended strongly on the sizes of the volumes considered and on the position of the measurement points and sound sources. This article considers the delimitation of the volumes not only virtually but physically using OSB panels. This is important given that in 
practice a floor will likely be partitioned, with a different insulation value for each part determined by the enclosed volume. Therefore, this analysis considered divided rooms to identify the extent of this difference in addition to the need to identify a procedure that would reduce the times of measurement; otherwise, it should measure the entire room divided into VVs (as was done in the previous article $\left.{ }^{9}\right)$. The results showed that both airborne sound insulation $\left(R^{\prime}\right)$ and impact sound level

( $L_{n}^{\prime}$ and $L_{n T}^{\prime}$ ) are influenced by the presence of mobile OSB walls in the case of volume $V_{2}$ : airborne sound insulation is lower and impact sound level is higher in the presence of the mobile walls. These differences derive from the differences in the values of the sound levels measured in the receiving rooms that cannot be attributed to the longer reverberation time observed without the mobile OSB walls. As the mobile OSB walls are used to bound both volumes, the differences are most likely mainly due to the different construction typologies of the boundary walls: for volume $V_{1}$, the walls are in most cases façades mainly made of glass, while in the case of volume $V_{2}$, there are also concrete stairwells. Therefore, it is assumed that the increase in sound levels in the presence of the mobile walls is due to reflections off them; the effects are more apparent near the walls. This was confirmed by analyzing separately the values of the levels and the SNQs obtained in positions near to and far from the mobile OSB walls. The values depended strongly on the measurement positions and source positions, and the differences between the measurements obtained in the presence and absence of the mobile walls were evident in the smaller analyzed volume $\left(V_{2}\right)$ where the differences are not compatible with the measurement uncertainty, as in volume $V_{1}$. For measurements in large, open rooms, it is therefore necessary to find new criteria for measurement; in particular, new restrictions on the minimum distances to be maintained between the microphones and between the microphones and the walls (as shown in the previous work ${ }^{9}$ ). To attribute properly the values of airborne sound insulation and impact sound level of a structure, particularly in the case of large, open rooms, it will be necessary to specify the size of the volumes in which the measurements are to be made and whether these volumes should be delimited virtually or actually. The analysis of the measurements also confirmed (as shown in the previous work ${ }^{9}$ ) that the SNQs $L_{n w}^{\prime}$ and $L_{n T w}^{\prime}$ in these applications are very different from each other and that a more suitable reference time $T_{0}$ is needed for the calculation of $L_{n T w}^{\prime}$ SNQ.

\section{Declaration of conflicting interests}

The author(s) declared no potential conflicts of interest with respect to the research, authorship, and/or publication of this article.

\section{Funding}

The author(s) received no financial support for the research, authorship, and/or publication of this article.

\section{References}

1. ISO 16283-1:2014. Acoustics - field measurement of sound insulation in buildings and of building elements - part 1: airborne sound insulation (International Organization for Standardization, 2014).

2. ISO 140:1998. Acoustics - measurement of sound insulation in buildings and of building elementspart 4: field measurements of airborne sound insulation between rooms (International Organization for Standardization, 1998); Part 5: field measurements of airborne sound insulation of facade elements and facades (International Organization for Standardization, 1998); Part 7: field measurements of impact sound insulation of building elements (International Organization for Standardization, 1998); Part 14: guidelines for special situations in the field, 2004 (Note: Withdrawn and replaced by Part 13).

3. Papadopoulos C. Development of an optimised, standard-compliant procedure to calculate sound transmission loss: design of transmission rooms. Appl Acoust 2002; 63: 1003-1029. 
4. Anderson JS, Bratos-Anderson M and Doany P. The acoustics of a large space with a repetitive pattern of coupled rooms. J Sound Vib 1997; 208(2): 313-329.

5. Farina A and Fornari G. Studio della Propagazione del Rumore in Ambienti Industriali Bassi e Vasti. In: Proceedings of the dBA-98, AUSL Modena, Italy, 16-18 September 1998.

6. Chiang W and Shu Y. Acoustical design of stages with large plane surfaces in rectangular recital halls. Appl Acoust 2003; 64: 863-884.

7. Jang HS, Kim YH and Jeon JY. Effects of stage volume and absorption on acoustics of concert halls. In: Proceedings of the 20th international congress on acoustics, Sydney, NSW, Australia, 23-27 August 2010. Australian Acoustical Society, NSW Division.

8. Zahorik P. Assessing auditory distance perception using virtual acoustics. J Acoust Soc Am 2002; 111(4): 1832-1846.

9. Zambon G, Angelini F, Belingheri A, et al. Case study: field measurements of sound insulation in large, open rooms. $J$ Build Acoust 2014; 21: 235-250.

10. UNI 8199:1998. Acoustic tests of air conditioning and ventilation (Ente Nazionale Italiano di Unificazione, 1998).

11. UNI 11347:2010. Acoustic classification of building units - procedure for assessment and verification in field (Ente Nazionale Italiano di Unificazione, 2010).

12. ISO 12999-1:2014. Acoustics - determination and application of measurement uncertainties in building acoustics - part 1: sound insulation (International Organization for Standardization, 2014).

13. Scrosati C, Scamoni F, Bassanino M, et al. Uncertainty analysis by a Round Robin Test of field measurements of sound insulation in buildings: single numbers and low frequency bands evaluation - airborne sound insulation. Noise Control Eng J 2013; 61(3): 291-306, http://dx.doi.org/10.3397/1/3761025

14. ISO 717:2013. Acoustics - rating of sound insulation in buildings and of building elements - part 2: impact sound insulation (International Organization for Standardization, 2013).

15. Farina A. RAMSETE - a new pyramid tracer for medium and large scale acoustic problems. In: Proceedings of the Euro-Noise 95, Lyon, France, 21-23 March 1995. France: CETIM.

16. Farina A. Verification of the accuracy of the pyramid tracing algorithm by comparison with experimental measurements of objective acoustic parameters. In: Proceedings of the ICA95, Trondheim, Norway, 26-30 June 1995. Trondheim, Norway: Acoustical Society of Norway. 